\title{
Nanomechanics of a magnetic shuttle device
}

\author{
S. I. Kulinich ${ }^{\text {a) }}$ \\ Department of Physics, University of Gothenburg, Göteborg SE-412 96, Sweden; B. Verkin Institute \\ for Low Temperature Physics and Engineering of the National Academy of Sciences of Ukraine, \\ 47 Lenin Ave., Kharkov 61103, Ukraine; and Department of Physics and Astronomy, \\ Seoul National University, Seoul 151-747, South Korea \\ L. Y. Gorelik \\ Department of Applied Physics, Chalmers University of Technology, Göteborg SE-412 96, Sweden
}

A. V. Parafilo

Department of Physics, University of Gothenburg, Göteborg SE-412 96, Sweden; B. Verkin Institute for Low Temperature Physics and Engineering of the National Academy of Sciences of Ukraine, 47 Lenin Ave., Kharkov 61103, Ukraine; and Department of Physics and Astronomy, Seoul National University, Seoul 151-747, South Korea

\section{R. I. Shekhter}

Department of Physics, University of Gothenburg, Göteborg SE-412 96, Sweden

Y. W. Park

Department of Physics and Astronomy, Seoul National University, Seoul 151-747, South Korea

M. Jonson

Department of Physics, University of Gothenburg, Göteborg SE-412 96, Sweden and SUPA, Institute of Photonics and Quantum Sciences, Heriot-Watt University, Edinburgh EH14 4AS, Scotland, United Kingdom (Submitted May 26, 2014)

Fiz. Nizk. Temp. 40, 1161-1169 (October 2014)

We show that self sustained mechanical vibrations in a model magnetic shuttle device can be driven by both the charge and the spin accumulated on the movable central island of the device. Different scenarios for how spin- and charge-induced shuttle instabilities may develop are discussed and shown to depend on whether there is a Coulomb blockade of tunneling or not. The crucial role of electronic spin flips in a magnetically driven shuttle is established and shown to cause giant magnetoresistance and dynamic magnetostriction effects. (C) 2014 AIP Publishing LLC.

[http://dx.doi.org/10.1063/1.4897412]

\section{Introduction}

An electric weak link, such as a point contact between two bulk conductors, ${ }^{1}$ dominates the electrical resistance of any device into which it is incorporated. A bias voltage applied across the device therefore creates an electric field that is strongest near the weak link, where the electrons can be greatly accelerated and gain considerable excess energy ("hot" electrons). ${ }^{2}$ Energy is pumped into the electronic subsystem only in a small volume with a linear dimension of the order of the length of the weak link, which can easily be much shorter than the characteristic length for energy transfer from the electrons to other degrees of freedom. In this case few excitations caused by electron energy relaxation are created in the vicinity of the weak link since most of the "hot" electrons have escaped well into the bulk conductors - and their density has decreased greatly-before they loose their excess energy. This means that the electrons can gain a large amount of extra energy, which can be fully controlled by the bias voltage applied, without any significant Joule heating of the device - a situation which has been widely exploited for point-contact spectroscopy. ${ }^{3}$ If, however, a certain type of elementary excitation is trapped within the weak link the situation can be drastically different. This is because even for a small excitation rate a significant amount of energy may then accumulate in the corresponding degree of freedom near the weak link and considerable "heating" of the subsystem corresponding to such a selected degree of freedom becomes possible.

Elementary excitations of lattice vibrations (phonons) are one of the most common results of inelastic relaxation of hot electrons in a weak link. ${ }^{4}$ If the excited phonons are free to propagate away form the vicinity of the link there is not much heating, provided the weak link is shorter than the phonon energy relaxation length. In this case electronphonon scattering gives rise to a small correction to the resistance of the device, which can be harvested by pointcontact spectroscopy to provide information about the phonon spectrum and the electron-phonon coupling strength in the material. ${ }^{3}$

A different situation occurs if localized vibrations of a mechanical resonator ("vibrons") can be excited. An example of such a resonator is the movable conducting "dot" of a nanomechanical shuttle device, suspended between bulk source and drain electrodes, ${ }^{5}$ which in effect serves as a 
weak electric link between the electrodes due to electron tunneling between dot and electrodes. Since the rate of electron tunneling is sensitive to the location of the dot the electronic and mechanical degrees of freedom are coupled and energy can be supplied to the latter if an electrical current is injected into the device. Rather than heating, the result of such an energy supply can-under the right circumstances, i.e., if the work done by the electrostatic force on the charged dot is positive over one vibration period-be an accumulation of coherent vibrons corresponding to self-excitation of centre-of-mass dot vibrations ${ }^{5}$ (for reviews see, e.g., Refs. 6 and 7).

The spin degree of freedom of the electrons affects the energy transfer to the vibronic subsystem in two ways if the source and/or drain electrodes of the shuttle device are made of magnetic material. First, and rather trivially, the tunneling rates will be spin-dependent if the electron densities of states in the electrodes are different for different spin projections. ${ }^{8}$ Secondly, and perhaps more interestingly, in addition to the electrostatic force that acts on the charged dot/shuttle it will be subject to a magnetic exchange force due to the coupling of the net spin of the dot to the magnetizations of the leads. Due to the interplay between the electrostatic Coulomb force and the spin-dependent exchange force a rich variety of electro-spintromechanical phenomena govern the operation of such a magnetic shuttle device. In this work we will study this interplay systematically in the limit when the source and drain electrodes are $100 \%$ spin polarized close to the Fermi level (i.e., we assume that they are ideal so-called "halfmetals" ${ }^{\prime \prime}$.

\section{Formulation of the problem}

Our model device, shown in Fig. 1, represents a standard shuttle device with a single spin nondegenerate electron energy level on the central island (to be referred to as the "quantum dot" or simply the "dot" in what follows) and electrons that are fully spin polarized along the magnetization in the magnetic source- and drain electrodes. The magnetization in the drain is assumed to be antiparallel to the one in the source, which leads to a spin-blockade of tunneling and a vanishing current in the zero temperature limit. Electron transport through the device is possible only if the spin of electrons on the quantum dot can be flipped by, e.g., an external magnetic field oriented perpendicularly to the

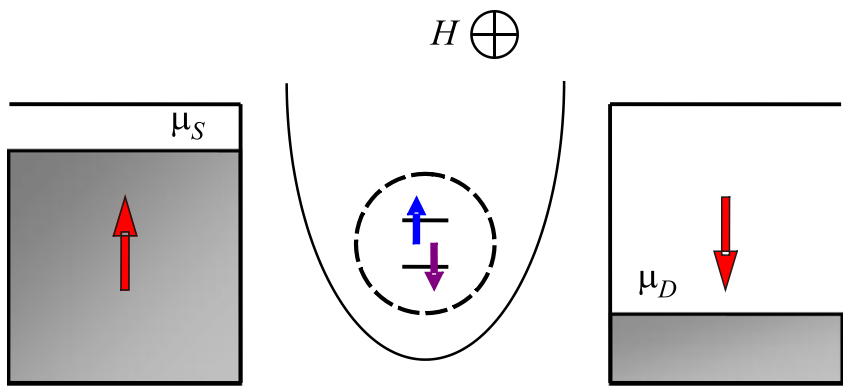

FIG. 1. Sketch of the nanomagnetic device discussed in the text: a movable quantum dot, modelled as a single spin nondegenerate electron level, is coupled to two leads with antiparallel magnetization and vibrates in the external harmonic potential. The potential difference $\mu_{S}-\mu_{D}=|e| V$ between the leads is due to a bias voltage $V$. An external magnetic field $H$ induces flips between the spin-up and spin-down states on the dot. magnetization of the leads. Accumulation of charge as well as spin on the dot are governed by the strength of the Coulomb- and spin-blockade phenomena making it possible for the nanomechanics of the device to be driven both by the electric field, which couples to the charge, and the magnetic exchange field, which couples to the spin. The Hamiltonian

$$
\hat{\mathcal{H}}=\hat{\mathcal{H}}_{l}+\hat{\mathcal{H}}_{d}+\hat{\mathcal{H}}_{v}+\hat{\mathcal{H}}_{t}
$$

of our system has four terms. The first term, $\hat{\mathcal{H}}_{l}$, describes noninteracting spin polarized electrons in the leads. The second term is the quantum dot Hamiltonian, $\hat{\mathcal{H}}_{d}$, which reads 9

$$
\begin{aligned}
\hat{\mathcal{H}}_{d}= & \varepsilon_{0}\left(a_{\uparrow}^{\dagger} a_{\uparrow}+a_{\downarrow}^{\dagger} a_{\downarrow}\right)-e \mathcal{E} x\left(a_{\uparrow}^{\dagger} a_{\uparrow}+a_{\downarrow}^{\dagger} a_{\downarrow}\right) \\
& -\frac{J_{S}(x)}{2}\left(a_{\uparrow}^{\dagger} a_{\uparrow}-a_{\downarrow}^{\dagger} a_{\downarrow}\right)-\frac{J_{D}(x)}{2}\left(a_{\downarrow}^{\dagger} a_{\downarrow}-a_{\uparrow}^{\dagger} a_{\uparrow}\right) \\
& -\frac{g \mu H}{2}\left(a_{\uparrow}^{\dagger} a_{\downarrow}+a_{\downarrow}^{\dagger} a_{\uparrow}\right)-U a_{\uparrow}^{\dagger} a_{\downarrow}^{\dagger} a_{\uparrow} a_{\downarrow} .
\end{aligned}
$$

Here the operator $a_{\sigma}^{\dagger}\left(a_{\sigma}\right)$ creates (annihilates) an electron on the dot with energy $\varepsilon_{0}$ and spin $\sigma=(\uparrow, \downarrow), x$ is the quantum dot displacement operator. The second term in Eq. (2) describes the coupling of the electron states in the dot with the electric field $(\mathcal{E})$, the third and fourth terms describe their coupling to the spin-polarized leads $\left(J_{j}(x)>0\right.$ is the strength of the ferromagnetic exchange coupling), the fifth term describes the coupling to the external magnetic field $H$ ( $\mu$ is the Bohr magneton, $g$ is the gyromagnetic ratio) and in the sixth term the intradot electron correlations are characterized by the Coulomb energy $U$.

Vibrations of the dot are described by the harmonic oscillator Hamiltonian

$$
\hat{\mathcal{H}}_{v}=\frac{p^{2}}{2 m}+\frac{m \omega^{2} x^{2}}{2},
$$

where $m$ is the mass and $\omega$ is the vibration frequency of the dot, $x$ is its coordinate and $p$ its canonical conjugated momentum; $[x, p]=i \hbar$.

The last term in our Hamiltonian (1) represents spinconserving tunneling of electrons between dot and leads,

$$
\hat{\mathcal{H}}_{t}=\sum_{k}\left(T_{S}(x) a_{k, S}^{\dagger} a_{\uparrow}+T_{D}(x) a_{k, d}^{\dagger} a_{\downarrow}\right)+\text { h.c. }
$$

Here $a_{k, j}^{\dagger}\left(a_{k, j}\right)$ is the electron creation (annihilation) operator for electrons with wave vector $k$ and spin up (down) for $j=S(D)$ (the spin index is suppressed), while $T_{j}(x)=T_{j}$ $\exp (j x / \lambda)$ is the position-dependent tunneling amplitude, $\lambda$ being the tunneling length and $j=(S, D)=(-1,+1)$. The electrons in each lead are held at a constant electrochemical potential $\mu_{S, D}=\mp e V / 2$ (relative to the Fermi level), where $V>0$ is the bias voltage. The electron density of states $\nu_{j}$ $=\nu$ in the leads is assumed to be independent of energy.

One needs to know the evolution of a reduced density matrix operator $\rho$, which describes the vibrational degree of freedom coupled to the electronic degrees of freedom of the single-level dot. The four possible electronic states are: $|0\rangle$, $|\uparrow\rangle=a_{\uparrow}^{\dagger}|0\rangle,|\downarrow\rangle=a_{\downarrow}^{\dagger}|0\rangle$, and $|2\rangle=a_{\downarrow}^{\dagger} a_{\uparrow}^{\dagger}|0\rangle$.

It is convenient to introduce dimensionless variables for time, $t \omega \rightarrow t$, dot displacement, $x / x_{0} \rightarrow x$ (where $x_{0}=$ $\sqrt{\hbar / m \omega}$ is the zero-point oscillation amplitude), tunneling 
length, $\lambda / x_{0} \rightarrow \lambda$, momentum, $p x_{0} / \hbar \rightarrow p$, and various characteristic energies, $\hbar \omega \rightarrow 1, e \mathcal{E} x_{0} / \hbar \omega \rightarrow d, g \mu H / \hbar \omega \rightarrow h$, $J(x) / \hbar \omega \rightarrow J(x), \quad \Gamma_{j}(x) / \omega \rightarrow \Gamma_{j}(x)\left(\hbar \Gamma_{j}(x)=2 \pi \nu\left|T_{j}(x)\right|^{2}\right.$ are partial level widths).

Following Ref. 10 one gets equations of motion for the reduced density matrix operators $\rho_{0} \equiv\langle 0|\rho| 0\rangle, \rho_{\uparrow} \equiv\langle\uparrow|\rho| \uparrow\rangle$, $\rho_{\downarrow} \equiv\langle\downarrow|\rho| \downarrow\rangle, \rho_{\uparrow \downarrow} \equiv\langle\uparrow|\rho| \downarrow\rangle$, and $\rho_{2} \equiv\langle 2|\rho| 2\rangle$ :

$$
\frac{\partial \rho_{0}}{\partial t}=-i\left[\hat{\mathcal{H}}_{v}, \rho_{0}\right]-\frac{1}{2}\left\{\Gamma_{S}(x), \rho_{0}\right\}+\sqrt{\Gamma_{D}(x)} \rho_{\downarrow} \sqrt{\Gamma_{D}(x)},
$$

$$
\begin{aligned}
\frac{\partial \rho_{\uparrow}}{\partial t}= & -i\left[\hat{\mathcal{H}}_{v}-x d, \rho_{\uparrow}\right]+\frac{i}{2}\left[J(x), \rho_{\uparrow}\right]-\frac{i h}{2}\left(\rho_{\uparrow \downarrow}-\rho_{\uparrow \downarrow}^{\dagger}\right) \\
& +\sqrt{\Gamma_{S}(x)} \rho_{0} \sqrt{\Gamma_{S}(x)}+\sqrt{\Gamma_{D}(x)} \rho_{2} \sqrt{\Gamma_{D}(x)},
\end{aligned}
$$$$
\frac{\partial \rho_{\downarrow}}{\partial t}=-i\left[H_{v}-x d, \rho_{\downarrow}\right]-\frac{i}{2}\left[J(x), \rho_{\downarrow}\right]
$$$$
+\frac{i h}{2}\left(\rho_{\uparrow \downarrow}-\rho_{\uparrow \downarrow}^{\dagger}\right)-\frac{1}{2}\left\{\Gamma_{+}(x), \rho_{\downarrow}\right\},
$$

$$
\begin{aligned}
\frac{\partial \rho_{2}}{\partial t}=-i & {\left[\hat{\mathcal{H}}_{v}-2 x d, \rho_{2}\right]+\sqrt{\Gamma_{S}(x)} \rho_{\downarrow} \sqrt{\Gamma_{S}(x)} } \\
- & \frac{1}{2}\left\{\Gamma_{D}(x), \rho_{2}\right\}, \\
\frac{\partial \rho_{\uparrow \downarrow}}{\partial t}= & -i\left[\hat{\mathcal{H}}_{v}-x d, \rho_{\uparrow \downarrow}\right]+\frac{i}{2}\left\{J(x), \rho_{\uparrow \downarrow}\right\} \\
& +\frac{i h}{2}\left(\rho_{\downarrow}-\rho_{\uparrow}\right)-\frac{1}{2} \rho_{\uparrow \downarrow}\left[\Gamma_{+}(x)\right] .
\end{aligned}
$$

In Eqs. (5)-(9) we have used the simplified notation $\Gamma_{j}(x)=\Gamma_{j} \exp (j 2 x / \lambda), \quad \Gamma_{+}(x)=\Gamma_{S}(x)+\Gamma_{D}(x)$, and $J(x)$ $=J_{S}(x)-J_{D}(x)$. In what follows we assume a linear $x$ dependence of $J(x): J(x)=J_{0}-\alpha x+\ldots, J_{0}=J_{S}(0)-J_{D}(0)$, $\alpha>0$.

Our operator equations contain terms that describe two mechanisms for the interaction between the vibrational and electronic subsystems, one electrical and one magnetic. In contrast to the electrical mechanism, the magnetic onewhich is due to the magnetic exchange force-is strongly connected to the spin dynamics. The result is a completely different dependence of the shuttle behavior on magnetic field.

We are interested in the classical motion of the dot and therefore use Eqs. (5)-(9) to derive the classical equations of motion for its coordinate and momentum. The result is

$$
\begin{gathered}
\frac{\partial x_{c}}{\partial t}=\operatorname{Tr}\left\{\frac{\partial}{\partial t}(x \rho)\right\}=p_{c}, \\
\frac{\partial p_{c}}{\partial t}=\operatorname{Tr}\left\{\frac{\partial}{\partial t}(p \rho)\right\} \\
=-x_{c}-d \operatorname{Tr}\left\{\rho_{0}-\rho_{2}\right\}-\alpha \operatorname{Tr}\left\{\rho_{\uparrow}-\rho_{\downarrow}\right\} .
\end{gathered}
$$

Therefore one needs to know the equations of motion for the zeroth moments, $R_{i}=\operatorname{Tr} \rho_{i}$.

The dynamics of the zeroth moments is coupled to the dynamics of the first moments. We will decouple at the level of the first moments by using the rule, $\operatorname{Tr} x \rho_{i}, \rightarrow x_{c} \operatorname{Tr} \rho_{i}$. In addition to restricting our study to the vibrational dynamics near the ground state we assume that the parameters $\left\{d, \alpha, \lambda^{-1}\right\}$ are small and linearize the problem with respect to the displacement $x_{c}$.

It is convenient to introduce linear combinations of $R_{i}$, so that

$$
\begin{aligned}
& R_{1,2}=\operatorname{Tr}\left\{\rho_{0} \pm \rho_{2}\right\}, \quad R_{3}=\operatorname{Tr}\left\{\rho_{\uparrow}-\rho_{\downarrow}\right\}, \\
& R_{4}=-i \operatorname{Tr}\left\{\rho_{\uparrow \downarrow}-\rho_{\downarrow \uparrow}\right\}, \quad R_{5}=\operatorname{Tr}\left\{\rho_{\uparrow \downarrow}-\rho_{\downarrow \uparrow}\right\} .
\end{aligned}
$$

Within these approximations the equations of motion for the zeroth moments are $\left(\Gamma_{ \pm}=\Gamma_{S} \pm \Gamma_{D}\right)$

$$
\begin{gathered}
\frac{\partial R_{2}}{\partial t}=-\frac{\Gamma_{+}}{2} R_{2}-\frac{\Gamma_{-}}{2}\left(1-R_{3}\right)+\frac{x}{\lambda}\left[\Gamma_{-} R_{2}+\Gamma_{+}\left(1-R_{3}\right)\right] \\
\frac{\partial R_{3}}{\partial t}=\frac{\Gamma_{-}}{2} R_{2}+\frac{\Gamma_{+}}{2}\left(1-R_{3}\right)-h R_{4} \frac{x}{\lambda}\left[\Gamma_{+} R_{2}+\Gamma_{-}\left(1-R_{3}\right)\right], \\
\frac{\partial R_{4}}{\partial t}=+h R_{3} \frac{\Gamma_{+}}{2} R_{4}+J_{0} R_{5}-\frac{x}{\lambda} \Gamma_{-} R_{2}-\alpha x R_{5} \\
\frac{\partial R_{5}}{\partial t}=-J_{0} R_{4}-\frac{1}{2} \Gamma_{+} R_{5}+\frac{x}{\lambda} \Gamma_{-} R_{5}+\alpha x R_{4} .
\end{gathered}
$$

(Note that the equation for $R_{1}$ is decoupled from the other equations and therefore not relevant in what follows.)

\section{Spintro- and electromechanics}

In this section we are interested in how the electrical and magnetic interaction mechanisms introduced above may induce a shuttle instability (or not) in two different regimes: with and without a Coulomb blockade of tunneling.

\subsection{Shuttle dynamics in the absence of a Coulomb blockade}

For small vibration amplitudes an analytical solution to the problem at hand can be found by perturbation theory in terms of the small parameters $\varepsilon=\left\{d, \alpha, \lambda^{-1}\right\}$. We solve the relevant equations by perturbation expansions,

$$
R_{i}(t)=R_{i}^{(0)}+R_{i}^{(1)}(t)+\cdots
$$

where $R_{i}^{(n)}$ is of $n$th order in $\varepsilon$. It is evident from Eqs. (13)-(16) that the functions $R_{i}^{(0)}$ do not depend on time. Hence,

$$
\begin{gathered}
R_{2}^{(0)}=-\frac{4 h^{2} \Gamma_{+} \Gamma_{-}}{\Delta}, \quad R_{3}^{(0)}=-\frac{\left(\Gamma_{+}^{2}+4 J_{0}^{2}\right)\left(\Gamma_{+}^{2}-\Gamma_{-}^{2}\right)}{\Delta}, \\
R_{4}^{(0)}=\frac{2 h \Gamma\left(\Gamma_{+}^{2}-\Gamma_{-}^{2}\right)}{\Delta}, \quad R_{5}^{(0)}=\frac{4 h J_{0}\left(\Gamma_{+}^{2}-\Gamma_{-}^{2}\right)}{\Delta},
\end{gathered}
$$

where

$$
\Delta=\left(\Gamma_{+}^{2}+4 J_{0}^{2}\right)\left(\Gamma_{+}^{2}-\Gamma_{-}^{2}\right) 4 h^{2} \Gamma_{+}^{2}
$$

It is convenient to define the vector-function $|\mathbf{R}\rangle=$ $\left(R_{2}^{(1)}, R_{3}^{(1)}, R_{4}^{(1)}, R_{5}^{(1)}\right)^{T}$. Then to first order in perturbation theory one has

$$
\frac{\partial|\mathbf{R}\rangle}{\partial t}=-\hat{A}|\mathbf{R}\rangle+x_{c}(t)\left(\lambda^{-1}\left|\mathbf{e}_{1}\right\rangle+\alpha\left|\mathbf{e}_{2}\right\rangle\right),
$$


where

$$
\hat{A}=\frac{1}{2}\left(\begin{array}{cccc}
\Gamma_{+} & -\Gamma_{-} & 0 & 0 \\
-\Gamma_{-} & \Gamma_{+} & 2 h & 0 \\
0 & -2 h & \Gamma_{+} & -2 J_{0} \\
0 & 0 & 2 J_{0} & \Gamma_{+}
\end{array}\right)
$$

and the vectors $\left|\mathbf{e}_{i}\right\rangle$ are defined as

$$
\begin{aligned}
& \left|\mathbf{e}_{1}\right\rangle=\frac{2\left(\Gamma_{+}^{2}-\Gamma_{-}^{2}\right)}{\Delta}\left(-2 h^{2} \Gamma_{+}, 0, h \Gamma_{+} \Gamma_{-},-2 h J_{0} \Gamma_{-}\right)^{T}, \\
& \left|\mathbf{e}_{2}\right\rangle=\frac{2 h\left(\Gamma_{+}^{2}-\Gamma_{-}^{2}\right)}{\Delta}\left(0,0,2 J_{0}, \Gamma_{+}\right)^{T} .
\end{aligned}
$$

Consequently, the eigenfrequencies of the shuttle vibrations can be found from the equation

$$
\begin{aligned}
& \frac{\partial^{2} x_{c}(t)}{\partial t^{2}}+x_{c}(t) \\
& \quad=-\int_{-\infty}^{t} d t^{\prime} x_{c}\left(t^{\prime}\right)\left\langle\mathbf { e } _ { 0 } \left|\mathrm{e}^{-\hat{A}\left(t-t^{\prime}\right)}\left(\lambda^{-1}\left|\mathbf{e}_{1}\right\rangle+\alpha\left|\mathbf{e}_{2}\right\rangle\right),\right.\right.
\end{aligned}
$$

where $\left|\mathbf{e}_{0}\right\rangle=(d, \alpha, 0,0)^{T}$.

At first, we consider the case when interaction between the electronic and mechanical degrees of freedom is only due to electrostatics $\left(\alpha, J_{0}=0 ; \mathrm{d} \neq 0\right)$. We assume that the tunneling coupling is symmetric $\left(\Gamma_{-}=0, \Gamma_{+}=2 \Gamma_{0}\right)$. The dispersion equation for the shuttle eigenfrequencies $\Omega$ is ${ }^{10}$

$$
\Omega^{2}-1=\frac{d}{\lambda}=\frac{2 \Gamma_{0} h^{2}}{\left(h^{2}+\Gamma_{0}^{2}\right)\left(\Gamma_{0}+i \Omega\right)} .
$$

We are interested in the sign of the imaginary part of the correction $\tilde{\omega}$ to the shuttle eigenfrequency, $\Omega=1+\tilde{\omega}$, which appears due to coupling with the leads. It follows from Eq. (23) that the imaginary part of eigenfrequency is always negative (and hence the amplitude increases with time), and therefore there is shuttle instability for any nonzero value of the external magnetic field.

In the opposite case, when the interaction between the electronic and vibrational degrees of freedom is only due to the magnetic exchange interaction between the leads and the $\operatorname{dot}\left(d=0 ; J_{0}, \alpha \neq 0\right)$, the dispersion equation for the shuttle vibration frequency takes the form

$$
\Omega^{2}-1=\frac{32 \alpha h^{2}}{\Delta} \frac{\Gamma_{+}^{2}-\Gamma_{-}^{2}}{D(\Omega)}\left(\Gamma_{+}+i \Omega\right)\left(\kappa-2 i \Omega \alpha J_{0}\right),
$$

where $\kappa=\left(2 \lambda^{-1}\right) J_{0}^{2} \Gamma_{-}-\alpha \Gamma_{+} J_{0}$, and

$D(\Omega)=\left(\Gamma_{+}+2 i \Omega\right)^{4}+\left(4 h^{2}+4 J_{0}^{2}-\Gamma_{-}^{2}\right)\left(\Gamma_{+}+2 i \Omega\right)^{2}-4 J_{0}^{2} \Gamma_{-}^{2}$.

In the limit of small tunneling asymmetry, $\Gamma_{-} \rightarrow 0$, Eq. (24) is simplified. The sign of the imaginary part of $\tilde{\omega}$ is determined by the sign of $J_{-}, \operatorname{sgn} \operatorname{Im} \Omega=\operatorname{sgn} J_{-}$. Therefore, if $J_{-}<0$ a shuttle instability occurs while if $J_{-}>0$ the stationary state of the dot is stable.

\subsection{Shuttle dynamics in the presence of a Coulomb blockade}

The equations of motion (5)-(9) for the reduced density matrix do not have any signature of Coulomb correlations. This is because they were obtained in the limit of high bias voltage, $e V / 2 \gg U$. Now we will consider the case $e V / 2$ $<U$, for which the Coulomb blockade is relevant. As shown in Ref. 10, the correct equations for the density matrix in the Coulomb blockade regime are obtained from Eqs. (5)-(9) by putting $\rho_{2}=0$ and by replacing $\Gamma_{+}(x)$ by $\Gamma_{D}(x)$.

By performing the same calculations as in Sec. 3.1 we can investigate the electro- and spintromechanical shuttle instability in the Coulomb blockade regime. ${ }^{11}$ In the absence of an exchange coupling with the leads $\left(\alpha, J_{0}=0 ; d \neq 0\right)$ we obtain the dispersion equation for a symmetric geometry $\left(\Gamma_{-}=0, \Gamma_{+}=2 \Gamma_{0}\right)$ as

$$
\Omega^{2}-1=-\frac{d}{\lambda} \frac{2 h^{2} \Gamma_{0}}{\Gamma_{0}^{2}+3 h^{2}} \frac{\Omega^{2}-h^{2}-i \Gamma_{0} \Omega}{\tilde{D}},
$$

where

$$
\tilde{D}=\frac{\Gamma_{0}}{2}\left(\Gamma_{0}^{2}+3 h^{2}-5 \Omega^{2}\right)+i \Omega\left(h^{2}+2 \Gamma_{0}^{2}-\Omega^{2}\right) .
$$

From Eq. (26) it follows that:

$$
\operatorname{Im} \tilde{\omega} \propto-\left\{\left(h^{2}-1+\Gamma_{0}^{2} / 2\right)^{2}+\Gamma_{0}^{2}\left(1-9 \Gamma_{0}^{2} / 16\right)\right\} .
$$

Therefore, for any magnetic field there is a shuttle instability if $\Gamma_{0}<4 / 3$. In the opposite case, $\Gamma_{0}>4 / 3$, there is a range of magnetic fields where a shuttle instability does not occur. For $\Gamma_{0} \gg 1$, this interval is $|h|<\Gamma_{0} / \sqrt{2}$, which implies that the shuttle regime of electron transport can not be realized in weak magnetic fields.

The conditions under which a shuttle instability occurs due to magnetic exchange forces only $\left(d=0 ; J_{0}, \alpha \neq 0\right)$ are determined by the equation

$$
\Omega^{2}-1=\frac{\alpha}{\lambda} \frac{h^{2} \Gamma_{0}^{2}}{\Gamma_{0}^{2}+3 h^{2}} \frac{i \Omega+\Gamma_{0}}{\tilde{D}},
$$

from which one finds that $\operatorname{Im} \tilde{\omega} \propto\left(h^{2}-3 \Gamma_{0}^{2}-3\right)$. It follows that the shuttle regime of transport corresponds to magnetic fields weaker than a certain critical value, $|h|<h_{c}$ $=\sqrt{3\left(\Gamma^{2}+1\right)}$.

It is clear from the results of this section that the conditions under which a shuttle instability and self sustained shuttle oscillations occur are quite complex. A physical interpretation of the results obtained must take the specific properties of the electrical- and magnetic-driven shuttle device into account together with the nature of the Coulomb correlations of electrons in the movable quantum dot. This will be the task of the next section.

\section{Spin-flip driven electromechanics}

In this section we will focus on a qualitative understanding of the basic physics that underlies the transduction of electrical energy from the battery, which maintains a voltage bias across the shuttle device, into mechanical energy stored in the shuttle vibrations. Whether energy is added to the mechanical vibrations or taken out of them depends on whether 
the mechanical work done by the total force that acts on the vibrating dot is positive or negative when averaged over one vibration period. The two contributions to this force considered here are the Coulomb force that couples to the net charge of the dot and the magnetic exchange force that couples to its total spin. Charge and spin on the dot vary as electrons tunnel between the dot and the leads, while the accumulated spin can also be changed without any change of charge if the spin of an electron can be flipped, e.g., by an external magnetic field.

We assume that the voltage bias is so large that the only contribution to the current is due to electrons tunneling first from the source electrode to the dot and then from the dot to the drain electrode (tunneling in the reverse direction from the dot to the source is blocked by the Pauli principle). Furthermore, we assume that the source and drain electrodes are $100 \%$ spin polarized with antiparallel magnetizations. The latter condition implies that electron transport through the device is blocked (in the low-temperature limit) in the absence of an external magnetic field that can flip the spin of electrons on the dot. To see this, note that electrons tunneling from the source to the drain are spin polarized (spin up, say) while there are only spin-down electron states available in the drain. Hence no current can flow through the device without spin flips on the dot.

In this situation $(H=0)$, let us assume that the dot vibrates without damping while carrying one spin-up electron as illustrated in Fig. 2(a). The charge as well as the spin are constants of motion since tunneling is blocked as described above. As a result the work done on the dot by the conservative Coulomb- and exchange forces averages to zero over one oscillation period and there is no energy transfer between the electronic and mechanical subsystems.

(a)

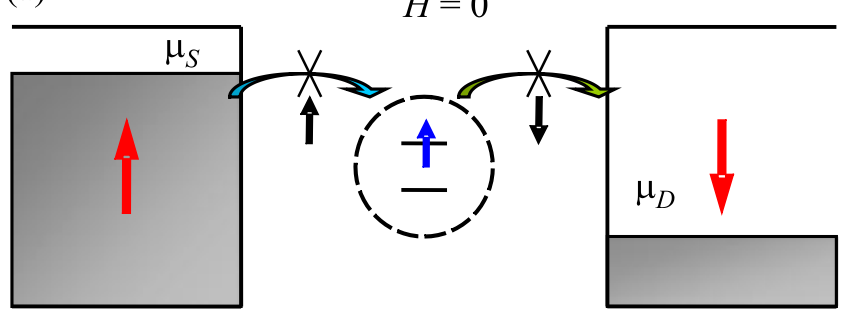

(b)

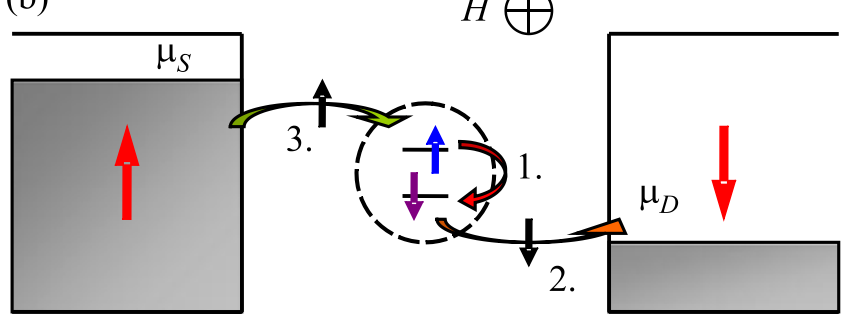

FIG. 2. (a) In the absence of an external magnetic field the current through the device is zero because spin-up electrons that enter the dot from the source electrode can not tunnel into the drain, where no empty spin-up states are available (spin blockade of tunneling). (b) An external magnetic field may flip the spin of the electron on the dot, which (i) opens up the possibility for this electron to tunnel to a spin-down state in the drain and hence for a current to flow and, independently, (ii) for a second (spin up) electron to enter the dot from the source (if Coulomb blockade effects can be neglected).
Only by switching on an external magnetic field, with a finite component $H$ perpendicular to the magnetization directions in the leads, can the spin be flipped from up to down so that tunneling from the dot to the drain becomes possible and a nonzero current can flow. It follows that the charge and spin population of the dot will vary with time and hence change both the Coulomb force and the exchange force acting on the dot. These changes will make the total work done by the force on the dot during one period of vibrational motion finite, corresponding to a finite energy transfer between the electronic and mechanical subsystems. Depending on whether the work done is positive or negative the dot will be accelerated or decelerated over an oscillation period. In the former case the initial equilibrium position of the dot will be unstable with respect to any mechanical displacement ("shuttle" instability) and self-sustained mechanical vibrations will develop, while in the latter case any spontaneous oscillation will be damped out.

The conditions under which a shuttle instability occurs crucially depend on whether the electronic tunneling events from the source to the dot on the one hand and from the dot to the drain on the other are correlated or not. If we are in the Coulomb blockade regime such correlations occur because double occupation of the dot (which is assumed to have a single spin-degenerate energy level) is prohibited. ${ }^{12}$ This means that a spin-up electron can tunnel from the source to the dot only after the one injected earlier has had its spin flipped and tunneled to the drain. The sequence of tunneling- and spin-flip events, which repeats itself to build up an electron current from the source to the drain, is illustrated for the case of weak magnetic field $H$ and large electron tunneling rates $\Gamma_{\mathrm{S}(D)}$ in Fig. 2(b).

In the absence of a Coulomb blockade the situation is completely different since tunneling events from the source and to the drain are then uncorrelated. The dot can be unpopulated or be populated by one spin-up electron, one spin-down electron or two electrons, one with spin up and one with spin down. Tunneling events will change the spin and charge on the dot, which will fluctuate randomly between the allowed configurations. The only remaining correlation in this case comes from the constraint that the time averaged current to and from the dot must be the same, which allows for finite time averaged spin and charge populations on the dot. Therefore the physics of the spintro-electro-mechanics should be discussed in terms of the probabilities for the various spin and charge configurations on the dot to occur.

At this point we note that both the tunneling of spin-up electrons from the source electrode to the dot and the tunneling of spin-down electrons from the dot to the drain increase the net spin (up) on the dot and can therefore be thought of as a source of spin-up electrons. This differs from the case of charge, where an electron that tunnels from the source to the dot increases the (negative) charge on the dot, while its charge is decreased when an electron tunnels from the dot to the drain. It turns out that this difference makes the spintromechanics of our device qualitatively different from its electro-mechanics, which relies entirely on the action of Coulomb forces. Below we will discuss this difference separately for the limits of strong Coulomb blockade and no Coulomb blockade. 


\subsection{Spintro- and electro-mechanical shuttling in the Coulomb blockade regime}

It is convenient to begin our analysis by neglecting the Coulomb force and focus on the role of the magnetic exchange force case. A particularly transparent picture of how the exchange force affects the shuttle vibrations emerges in the limit of weak magnetic field $H$ and large electron tunneling rates $\Gamma_{S(D)}$. In this limit, where $\Gamma_{S} \gg \omega \gg$ $(\mu H / \hbar)^{2} / \Gamma_{D}$ and $\omega / 2 \pi$ is the natural vibration frequency of the dot, we note that transport in the Coulomb blockade regime is determined by the sequence of electron tunnelingand spin-flip events shown in Fig. 2(b). Flipping the spin of a spin-up electron on the dot is a prerequisite for its ability to tunnel to the drain electrode, an event which in its turn changes the net charge and spin on the dot and allows another spin-up electron to tunnel to the dot from the source. To proceed, let us first elaborate somewhat our arguments why no total work is done by the exchange force $F$ as the dot vibrates under the influence of an elastic force only. In the absence of an external magnetic field ${ }^{13}$ the dot is in this case occupied by a spin-up electron emanating from the source electrode. This spin is a constant of motion and hence no electrical current through the device is possible since only spin-down states are available in the drain electrode. During the oscillatory motion of the dot the exchange force is therefore always directed towards the source electrode while its magnitude only depends on the position of the dot, $F=F_{0}(x)$. As a result, no net work is done by the exchange force on the dot. This is because contributions are positive or negative depending on the direction of the dot's motion and cancel when summed over one oscillation period. A finite amount of work can only be done if the exchange force deviates from $F_{0}(x)$ as a result of spin-flip processes induced by the external magnetic field. Such a deviation can be viewed as an additional random force $F_{H}$ that acts in the opposite direction to $F_{0}(x)$. In the limit of large tunneling rate, $\Gamma_{S(D)} \gg \mu H / \hbar$, and small vibration amplitude a spin flip occurs with a probability $\propto(\mu H / \hbar)^{2} /\left(\omega \Gamma_{D}\right)$ during one oscillation period and is instantly ${ }^{14}$ accompanied by the tunneling of the dot electron into the drain electrode, thereby triggering the force $F_{H}$. The duration of this force is determined by the time $\delta t \sim$ $1 / \Gamma_{S}(x(t))$ it takes for the spin of the dot to be "restored" by another electron tunneling from the source electrode.

The spin-flip induced random force $F_{H}=-F_{0}(x)$ is always directed towards the drain electrode. Hence, its effect depends on the dot's direction of motion: as the dot moves away from the source electrode it will be accelerated, while as it moves towards the source it will be decelerated. Since a spin flip may occur at any point on the trajectory one needs to average over different spin-flip positions in order to calculate the net work done on the dot. The result, which depends on the competition between the effect of spin flips that occur at the same position but with the dot moving in opposite directions, is nonzero because $\delta t$ is different in the two cases. As the dot moves away from the source electrode the tunneling rate to this electrode will decrease while as the dot moves towards the source it will increase. This means that the duration of spin-flip induced acceleration will prevail over the one for deceleration. As a result, in weak magnetic fields, the dot will accelerate with time and one can expect a spintro-mechanical shuttle instability in this limit.
The situation is qualitatively different in the opposite limit of strong magnetic fields, where $\Gamma_{S(D)} \ll \mu H / \hbar$ and the spin rotation frequency therefore greatly exceeds the tunneling rates. In this case the quick precession of the electron spin in the dot averages the exchange force to zero if one neglects the small effects of electron tunneling to and from the dot. If one takes corrections due to tunnelling into account (having in mind that the source electrode only supplies spin-up electrons) one comes to the conclusion that the average spin on the dot will be directed upwards. This results in a net spintro-mechanical force in the direction opposite to that of the net force occurring in a weak magnetic field limit. As a result, in strong magnetic fields one expects on the average a deceleration of the dot. Therefore, there will be no shuttle instability for such magnetic fields.

As we have discussed above spin-flip assisted electron tunneling from source to dot to drain in our device results in a magnetic exchange force that attracts the dot to the source electrode. It is interesting to note that this is contrary to the effect of the Coulomb force in the same device. Indeed, since the Coulomb force depends on the electric charge of the dot it repels the dot from the source electrode. Hence, while the dot is empty as the result of a spin-flip assisted tunneling event from dot to drain, an "extra" attractive Coulomb force $F_{Q}$ is active. An analysis fully analogous with our previous analysis of the "extra" repulsive magnetic exchange force $F_{H}$ leads to the conclusion that the effect of the Coulomb force will be just the opposite to that of the exchange force. If the exchange force is sufficiently weak, this means that in the Coulomb blockade regime there is no shuttle instability in the limit of weak magnetic field, while in strong magnetic fields electron shuttling occurs. Our full analysis in Sec. 3.2 confirms the predictions here made for some limiting cases using only qualitative arguments.

\subsection{Spintro- and electro-mechanical shuttling in the absence of a Coulomb blockade}

Here we will begin our analysis of the magnetic shuttle device by neglecting the magnetic exchange force and only take the Coulomb force on the dot into account. This implies that the only effect of the electron spin on the electromechanics of our device is to block the tunneling of spin-up electrons to the drain electrode. The Coulomb force couples to the charge of the dot, which (measured in units of the electron charge) is a monotonically decreasing function of the distance between the dot and the source electrode as shown in Fig. 3(a). The bold line in the figure corresponds to the limit of an adiabatically slow motion of the dot so that for each position of the dot there is ample time to for the charge on the dot to adjust to the value that makes the current from the source equal to the current to the drain. In this case the charge on the dot depends on its position but not on the direction of motion of the vibrating dot. The positive work done by the Coulomb force on the dot as it moves in one direction will therefore be exactly cancelled by the negative work it does when the dot is moving in the opposite direction.

A finite amount of work can only come from nonadiabatic corrections to this picture. These arise when the charge of the dot at a certain position does not have time to fully adjust to the "adiabatic" value at that point but remains at a 
(a)
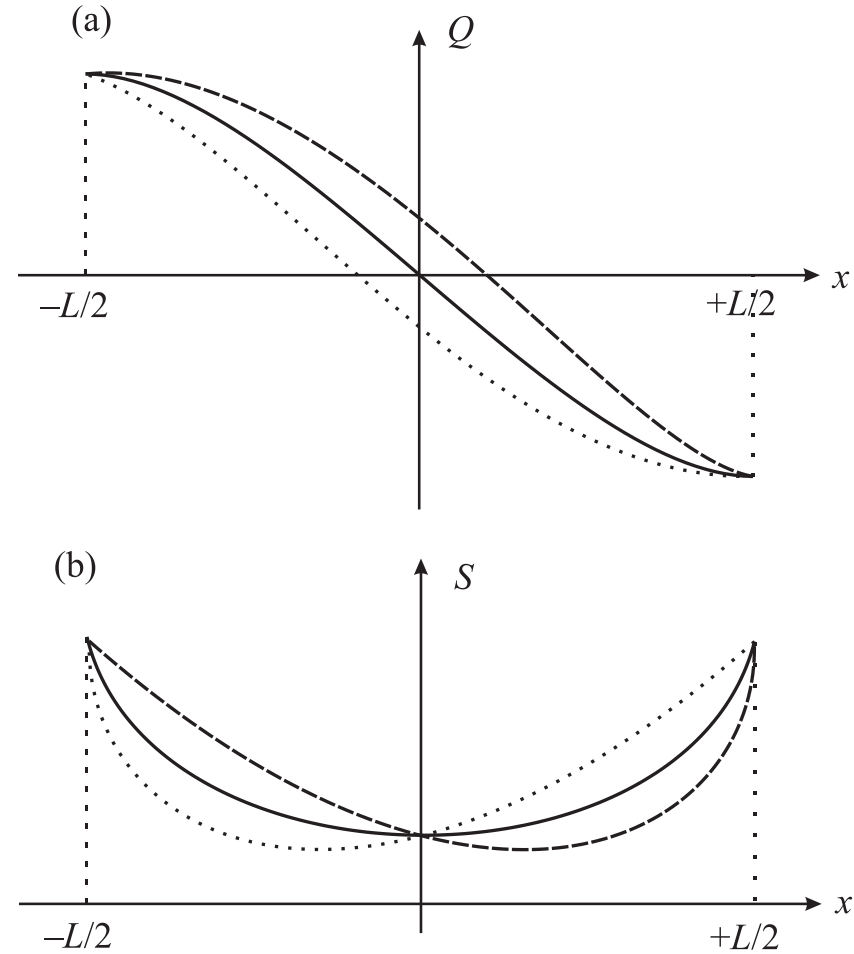

FIG. 3. (a) Schematic plot of the accumulated charge on the quantum dot as a function of its position in the limit when the vibrational motion of the dot is adiabatically slow (solid line). Corrections to this adiabatic result are due to retardation effects and depend on whether the dot is moving away from (dashed curve) or towards (dotted curve) the source electrode. (b) Total spin accumulated on the quantum dot in the adiabatic regime as the function of the dot displacement (solid line). The dotted and dashed lines indicate the nonadiabatic corrections to the value of the averaged spin.

value that it would have had at an earlier time if the motion had been adiabatic. This "retardation effect" gives a dot charge that depends on the direction of motion-larger than the adiabatic value when motion is in the direction of the drain, smaller when the dot moves in the direction of the source as illustrated by the dashed and dotted curves in Fig. 3(a). Since a larger (smaller) amount of charge on the dot increases (decreases) the repulsive Coulomb force between the dot and the source electrode, it is clear from Fig. 3 that the vibrational motion of the dot will be accelerated by the Coulomb force. In other words, positive work will be done on the dot with the result that its kinetic energy will increase monotonically as will its oscillation amplitude and we have an electromechanical shuttle instability.

We conclude that without a Coulomb blockade the Coulomb force leads to a shuttle instability for any value of the magnetic field strength if more energy is pumped into the shuttle motion than is lost to the environment by dissipation.

Now let us consider the opposite limit of "spintromechanics," where we neglect the Coulomb force and only consider the effect of the magnetic exchange force. In this case the total spin accumulated on the dot determines the force responsible for the transfer of energy between the electronic and mechanical subsystems. The controlling factors turn out to be the transfer rates of spin-up electrons from the source and spin-down electrons to the drain. From the point of view of spin (but not charge) it is convenient to consider the latter process as a transfer of spin-up electrons from the drain to the dot. In this view both the source electrode and the drain electrode act as sources of spin-up electrons. Since the tunneling rate increases as the dot approaches either electrode, one expects the total spin accumulated on the dot in the adiabatic limit to be a nonmonotonic function of the dot's position with a minimum when the dot is at the centre of the device (see the solid curve in Fig. 3(b)). As the accumulated spin will not depend on the direction of the dot's motion we can again argue that the net work done by the exchange force will average to zero over one oscillation period in this case.

As in the case of the Coulomb force, which we discussed in the first part of this subsection, the exchange force will only do finite work on the dot if nonadiabatic corrections to the spin accumulation are considered. Using the same arguments as before one finds that these qualitatively correspond to the dashed (dot moving away from the source) and dotted (dot moving towards the source) curves in Fig. 3(b). The results is that a finite amount of work may be done (see below) on the dot during one oscillation period.

In order to determine the value and sign of the work done by the exchange force one has to take into account the strength of the exchange interaction between the dot and the two electrodes, which always have opposite signs. It is straightforward to see that if the magnetization of the source and drain electrodes have equal magnitude (but opposite directions), symmetry considerations will lead to a net work that is zero even in the nonadiabatic case (see Fig. 3(b)). The interesting conclusion is that in contrast to the electrically driven symmetric shuttle a spin-flip driven symmetric magnetic shuttle does not have a shuttle instability.

If the magnitude of the antiparallel magnetizations of the source- and drain electrodes are different, however, energy can be pumped into or out of the mechanical subsystem depending on which electrode has the largest magnetization. Using similar arguments as above one finds that if the magnetic coupling to the source electrode dominates then negative work is done on the vibrating dot and there is no shuttle instability. If, on the other hand, coupling to the drain electrode dominates then energy is pumped into the mechanical vibrations resulting in a shuttle instability.

The qualitative picture presented here for the electrically and magnetically driven shuttles in the limits of strong and weak Coulomb blockade fully correspond to the results obtained by the rigorous analysis described in Secs. 2 and 3.

\section{Conclusions}

In this work we have explored the possibility for the electronic spin to contribute to the electromechanics of a magnetic shuttle device. A number of new functionalities can be achieved by exploiting the magnetic exchange force on the movable dot at the center of the device in addition to the electrostatic Coulomb force. The exchange force is determined by the spatial dependence of the interaction of the electronic spin accumulated on the dot with the magnetized leads while the Coulomb force is due to the interaction between the charge on the dot and the electric field caused by a voltage bias between the source- and drain electrodes.

The possibility to switch the sign of the electric charge on the movable dot in tact with the change of direction of the dot's motion - as electrons are sequentially loaded onto the dot from the source and off-loaded to the drain-leads to 
a shuttle instability in the standard (electrically driven) shuttle. In contrast, it is necessary to apply an external magnetic field (perpendicular to the antiparallel magnetizations in the leads) to switch the sign of the accumulated spin (by magnetically induced spin flips). We have shown that such spin-flip induced magnetic driving of the mechanical vibrations of the dot may also result in a shuttle instability.

In an electrically (charge) driven shuttle the nanomechanics is controlled by the electric field via the applied bias voltage. In contrast, the spin-flip induced nanomechanics of a magnetic shuttle is controlled by the external magnetic field through a "dynamical magnetostriction" effect, which also serves as a new "mechanical" mechanism for giant magnetoresistance. The crucial sensitivity of this magnetoresistance to the strength of the Coulomb blockade phenomenon (discussed in Sec. 4) should make it possible to realize a magnetic shuttle device with electric-gate controlled giant magnetoresistance.

The relative strength of the Coulomb force and the exchange force can be controlled by the externally applied driving voltage and magnetic field. This means that by varying these fields one can switch a situation where the electrical force is dominating to one where the magnetic force determines the operation of the device. This is important for magneto-electric transduction and is based on the comparable strengths of the Coulomb force and the exchange force in realistic tunnel devices (see Ref. 9).

Another source of electronic spin flips is spin-flip assisted resonant absorption of microwave radiation. This phenomenon is somewhat similar to that of microwave induced electronic intermode transitions in quantum point contact $^{15,16}$ in the sense that electronic spin-flip transitions occur selectively at certain values of the dot displacement. Typical values of the exchange energy correspond to temperatures of a few kelvin and a frequency domain in the far infrared region which is important for applications. Microwave properties of magnetic shuttle devices will be the subject of a separate publication.

Financial support from the Swedish VR and the Leading Foreign Research Institutes Recruitment Program (200900514) of NRF, Korea, is gratefully acknowledged. S.K. and
A.P. acknowledge financial support from the National Academy of Science of Ukraine (Grant No. 4/14-N). S.K. and A.P. thank the Dept. of Physics at the University of Gothenburg and the Dept. of Physics and Astronomy at Seoul National University for their hospitality.

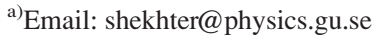

${ }^{1}$ R. Holm, Electrical Contacts, 3rd ed. (Springer-Verlag, Berlin, 1958).

${ }^{2}$ A. N. Omelyanchouk, I. O. Kulik, and R. I. Shekhter, JETP Lett. 25, 437 (1977); I. O. Kulik, A. N. Omelyanchouk, and R. I. Shekhter, Fiz. Nizk. Temp. 3, 1543 (1977) [Sov. J. Low Temp. Phys. 3, 740 (1977)].

${ }^{3}$ Yu. G. Naidyuk and I. K. Yanson, Point-Contact Spectroscopy, Springer Series in Solid State Sciences Vol. 145 (Springer, New York, 2005).

${ }^{4}$ I. K. Yanson, Zh. Eksp. Teor. Fiz. 66, 1035 (1974) [Sov. Phys. JETP 39, 506 (1974)].

${ }^{5}$ L. Y. Gorelik, A. Isacsson, M. V. Voinova, B. Kasemo, R. I. Shekhter, and M. Jonson, Phys. Rev. Lett. 80, 4526 (1998).

${ }^{6}$ R. I. Shekhter, Y. Galperin, L. Y. Gorelik, A. Isacsson, and M. Jonson, J. Phys.: Condens. Matter 15, R441 (2003); R. I. Shekhter, L. Y. Gorelik, M. Jonson, Y. M. Galperin, and V. M. Vinokur, J. Comput. Theor. Nanosci. 4, 860 (2007)

${ }^{7}$ R. I. Shekhter, L. Y. Gorelik, I. V. Krive, M. N. Kiselev, A. V. Parafilo, and M. Jonson, Nanoelectromech. Syst. 1, 1 (2013); R. I. Shekhter, L. Y. Gorelik, I. V. Krive, M. N. Kiselev, S. I. Kulinich, A. V. Parafilo, K. Kikoin, and M. Jonson, Fiz. Nizk. Temp. 40, 775 (2014) [Low Temp. Phys. 40, 7 (2014).

${ }^{8}$ Collosal Magnetoresistive Oxides, edited by Y. Tokura (Gordon and Breach, New York, 2000).

${ }^{9}$ R. I. Shekhter, A. Pulkin, and M. Jonson, Phys. Rev. B 86, 100404(R) (2012).

${ }^{10}$ D. Fedorets, L. Y. Gorelik, R. I. Shekhter, and M. Jonson, Phys. Rev. Lett. 95, 057203 (2005); L. Y. GorelikD. Fedorets, R. I. Shekhter, and M. Jonson, New J. Phys. 7, 242 (2005)

${ }^{11}$ S. I. Kulinich, L. Y. Gorelik, A. N. Kalinenko, I. V. Krive, R. I. Shekhter, Y. W. Park, and M. Jonson, Phys. Rev. Lett. 112, 117206 (2014).

${ }^{12}$ L. Y. Gorelik, S. I. Kulinich, R. I. Shekhter, M. Jonson, and V. M. Vinokur, Phys. Rev. Lett. 95, 116806 (2005).

${ }^{13}$ For the moment we also neglect mechanical damping and intrinsic spinflip mechanisms.

${ }^{14}$ The first nonvanishing term in a perturbation expansion of the tunneling probability is of order $\left(\mu H / \Gamma_{D}\right)^{2}$ and corresponds to spin-flip assisted tunneling.

${ }^{15}$ A. Grincwajg, L. Y. Gorelik, V. Z. Kleiner, and R. I. Shekhter, Phys. Rev. B 52, 12168 (1995).

${ }^{16}$ L. I. Glazman and R. I. Shekhter, Solid State Commun. 66, 65 (1988).

This article was published in English in the original Russian journal. Reproduced here with stylistic changes by AIP Publishing. 